\title{
Efficacy of L-carnitine supplementation on frailty status and its biomarkers, nutritional status, and physical and cognitive function among prefrail older adults: a double-blind, randomized, placebo- controlled clinical trial
}

This article was published in the following Dove Press journal:

Clinical Interventions in Aging

17 November 2016

Number of times this article has been viewed

\author{
M Badrasawi ${ }^{1,2}$ \\ Suzana Shahar' \\ AM Zahara' \\ R Nor Fadilah ${ }^{3}$ \\ Devinder Kaur Ajit Singh ${ }^{4}$ \\ 'Dietetic Programme, School of \\ Healthcare Sciences, Universiti \\ Kebangsaan Malaysia, Kuala Lumpur, \\ Malaysia; ${ }^{2}$ Nutrition Program, Faulty \\ of Applied Sciences, Palestine \\ Polytechnic University, Hebron, \\ Palestine; ${ }^{3}$ Biomedical Programme, \\ School of Healthcare Sciences, \\ Universiti Kebangsaan Malaysia, Kuala \\ Lumpur, Malaysia; ${ }^{4}$ Physiotherapy \\ Programme, School of Rehabilitation \\ Sciences, Universiti Kebangsaan \\ Malaysia, Kuala Lumpur, Malaysia
}

Background: Frailty is a biological syndrome of decreased reserve and resistance to stressors due to decline in multiple physiological systems. Amino acid deficiency, including L-carnitine, has been proposed to be associated with its pathophysiology. Nevertheless, the efficacy of L-carnitine supplementation on frailty status has not been documented. Thus, this study aimed to determine the effect of 10-week L-carnitine supplement (1.5 g/day) on frailty status and its biomarkers and also physical function, cognition, and nutritional status among prefrail older adults in Klang Valley, Malaysia.

Methodology: This study is a randomized, double-blind, placebo-controlled clinical trial conducted among 50 prefrail subjects randomized into two groups (26 in L-carnitine group and 24 in placebo group). Outcome measures include frailty status using Fried criteria and Frailty Index accumulation of deficit, selected frailty biomarkers (interleukin-6, tumor necrosis factoralpha, and insulin-like growth factor-1), physical function, cognitive function, nutritional status and biochemical profile.

Results: The results indicated that the mean scores of Frailty Index score and hand grip test were significantly improved in subjects supplemented with L-carnitine $(P<0.05$ for both parameters) as compared to no change in the placebo group. Based on Fried criteria, four subjects (three from the L-carnitine group and one from the control group) transited from prefrail status to robust after the intervention.

Conclusion: L-carnitine supplementation has a favorable effect on the functional status and fatigue in prefrail older adults.

Keywords: frailty, Fried criteria, Frailty Index accumulation of deficit, L-carnitine supplementation, prefrail elderly, physical function, frailty biomarkers, randomized controlled clinical trial

\section{Background}

Aging is associated with functional decline, loss of autonomy, and a wide spectrum of adverse outcomes as mooted in literature. ${ }^{1}$ Due to a global rise in life expectancy, maintaining physical function and preventing disability in advanced age are among the major clinical and public health priorities. ${ }^{2,3}$ Frailty is one of the main factors that suppresses successful aging and leads to adverse health consequences, mainly falls and delirium after minor stressor. ${ }^{4}$
Correspondence: Suzana Shahar
Dietetics Programme, School of Healthcare Sciences, Faculty of Health Sciences, Universiti Kebangsaan Malaysia, Jalan Raja Muda Abdul Aziz, 50300 Kuala Lumpur, Malaysia

Tel +60 392897194

Fax +60 32693 8717

Email suzana.shahar@ukm.edu.my 
The concept of frailty can be seen as a multidimensional, transitional state of increased vulnerability and loss of the ability to adapt to stress. ${ }^{5}$ It has also been recognized as a distinct clinical syndrome of cumulative decline in many physiological systems during lifetime ${ }^{6,7}$ with a biological basis. ${ }^{8}$ Given this recognition, numerous physiological systems have been studied in the light of their relationship with frailty. ${ }^{9}$ Among these physiological systems are inflammation, neuromuscular dysfunction, endocrine dysregulation, immune dysfunction, abnormalities in energy metabolism, ${ }^{9}$ and central nervous system failure. ${ }^{10}$

L-carnitine ( $\beta$-hydroxy- $\gamma$-N-trimethylamino-butyric acid) is an essential dietary nutrient, which can be synthesized endogenously. ${ }^{11}$ L-carnitine is a metabolically important compound, ${ }^{11,12}$ which has several functions in the human body. ${ }^{8}$ The main function of L-carnitine in cellular energy metabolism is as a requisite in the transport of the long chain fatty acids to the inner mitochondrial membrane and the site of beta oxidation. ${ }^{11}$ The other functions of L-carnitine include buffering of mitochondrial acyl $\mathrm{CoA} / \mathrm{CoA}$ ratio, oxidation of branched amino acids, and having protective effects on the endogenous antioxidant defense system. ${ }^{13}$

Clinically, L-carnitine is important for fatty acid metabolism, as a antiradical agent, and for prevention against oxidative damage. ${ }^{11}$ L-carnitine supplementation has been studied in a few diseases and in adults with L-carnitine deficiency, such as in cancer, HIV, and kidney failure patients in intervention studies. The supplementation showed a significant favorable effect on cancer-related anorexia, ${ }^{14}$ cachexia, ${ }^{15}$ and fatigue. ${ }^{15-17}$ It is also known to have an enhancing metabolic and immunological effect on HIV patients ${ }^{18,19}$ and increased muscle strength in kidney failure patients. ${ }^{20}$

Among older adults, L-carnitine supplementation was beneficial in improving physical, mental, and cognitive function. ${ }^{21}$ Improvement in body composition and fatigue has also been reported with L-carnitine supplementation. ${ }^{22}$ Positive effects on mood and cognitive functions were demonstrated following a 7-week supplementation of L-carnitine among older adults with depression. ${ }^{23}$

Aging-related changes in biological system, particularly the mitochondria dysfunction, suggest that L-carnitine deficiency may be associated with aging and age-related disorders. A relationship between L-carnitine status and frailty was reported in a study by Crenstil, ${ }^{9}$ with L-carnitine deficiency deduced to related pathophysiological changes in frailty, such as neuromuscular abnormalities, inflammation, decrease of energy production, and immune impairment. ${ }^{7}$
However, more information regarding the relationship between L-carnitine and frailty is required. The same urge is needed to conduct intervention and experimental studies using L-carnitine supplementation among frail elderly people. Up to date, the published studies that have investigated the effectiveness of L-carnitine on frailty are sparse. Therefore, the aim of the present study was to determine the effectiveness of L-carnitine supplementation on frailty status among Malaysian older adults with prefrailty.

\section{Methodology}

This study is a double-blind, randomized, placebo-controlled clinical trial with L-carnitine supplementation among prefrail older adults. The primary outcomes of the intervention were frailty status, frailty biomarkers, and physical function, while the secondary outcomes included nutritional status, body composition, and cognitive function. The study protocol was approved by the Department of Social and Welfare (December 13, 2013) and Universiti Kebangsaan Malaysia Medical Research and Ethics Committee (UKM 1.5.3.5/244/NN-149-2013). All patients provided written informed consent. The intervention was carried out from March 23 to July 14, 2014. The recruited subjects were assessed three times through the intervention period at baseline, week 5, and week 10. Figure 1 summarizes the subjects' recruitment in a flowchart. The sample size was calculated using Chan formula for sample size calculation in randomized controlled clinical trials. ${ }^{24}$ The mean and standard deviations were taken from a similar randomized clinical trial Rondanelli et $\mathrm{al}^{25}$ conducted to determine the improvement of the hand grip as a primary outcome among elderly individuals using amino acid supplementation. It showed significant changes in the mean of the hand grip among the intervention group. Ninety percent power and 0.05 level of confidence were assumed to calculate the sample size. The final sample size calculation deemed required was 25 subjects in each group.

\section{Subjects' selection}

This study is a part of a larger nationwide longitudinal study on neuroprotective model for healthy longevity among Malaysian older adults as reported earlier. ${ }^{26}$ This present study was conducted at the state of Selangor in Malaysia. Subjects were selected through a multistage random sampling in order to assure the representativeness of the three major community ethnic groups in Malaysia (Malay, Chinese, and Indian) from ten different districts. The chosen districts were with elderly population making up at least $10 \%$ of 


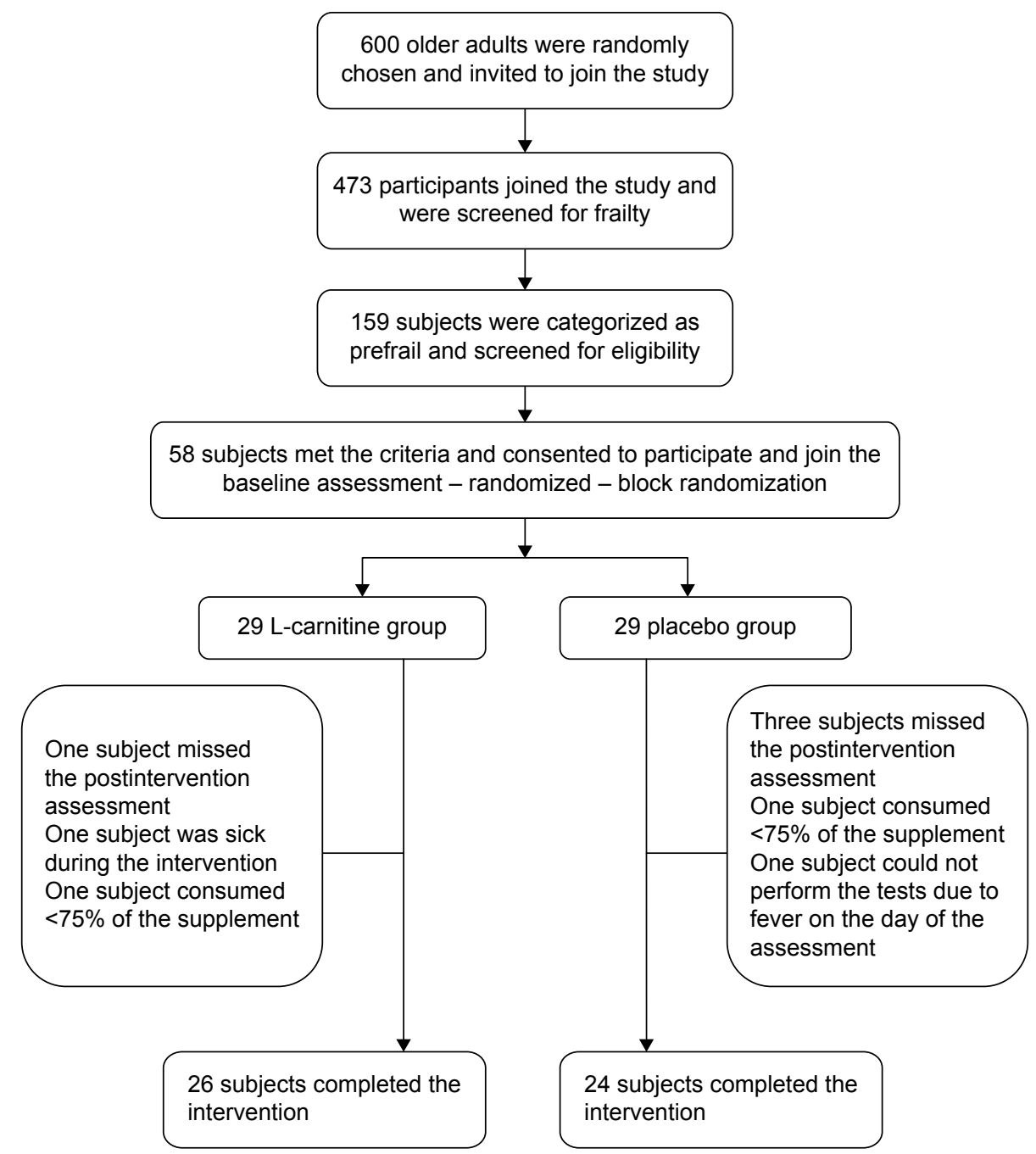

Figure I Subjects' recruitment flowchart.

the total population according to Malaysian Department of Statistics. More than 600 community dwelling individuals aged 60 years and above, with no known terminal and mental illnesses, were invited to participate in a health screening. Out of the 473 subjects consented and participated in the screening, 159 subjects were categorized as prefrail according to Fried criteria. ${ }^{27}$ These participants were then shortlisted according to the study criteria. Subjects who had any of the following were excluded: uncontrolled diabetes mellitus (glycated haemoglobin $\left[\mathrm{HbA}_{1 \mathrm{c}}\right]>7.5 \%$ ), cognitive impairment (Mini-Mental State Examination $[\mathrm{MMSE}]<18$ ), being malnourished with muscle wasting, and taking L-carnitine supplements for the past year prior to the study. A total of 58 subjects who consented to join the study were randomized into two groups using block randomization stratified by sex. Code numbers were given to the subjects and written on folded papers for randomized selection. Randomized selection of the numbers were done by one of the research team members who was totally uninvolved in this study.

\section{Preparation of the supplements}

L-carnitine supplement (500 $\mathrm{mg} / \mathrm{cap}$ ) was purchased from Cambert (M) Sdn Bhd, (Selangor, Malaysia). They had been repacked a day before the intervention in dark white colored pharmaceutical bottles to assure blinding. The placebo capsules (corn starch filling) were made at the pharmaceutical laboratory, Faculty of Pharmacy, UKM, Kuala Lumpur campus, by a trained pharmacist using a capsule filling machine, Product type: JCT. The placebo capsules were prepared 3 weeks before the intervention, and packed in the same bottles that the L-carnitine supplements were repacked in. The placebo capsules were completely identical when compared 
with the L-carnitine supplement in terms of color, size, and appearance. However, the only difference was in the weight; the placebo capsules weight range was from 520 to $550 \mathrm{mg}$, while the L-carnitine capsules weight ranged from 600 to $610 \mathrm{mg}$. It was difficult to recognize the difference in the weight by visual observation.

\section{Intervention and ensuring compliance}

Detailed briefing about the study and the benefits of the intervention was given to the subjects at the baseline assessment. All subjects were informed that they had to take the supplementation as prescribed on the bottles (one capsule [500 mg], tid, for 10 weeks), and their participation was optional. Each subject was given a booklet, including a timetable of consuming the supplement. Participants were requested to fill in daily the number and time of the capsules they took. On the follow-up assessments, the supplementation bottles were collected from the subjects in each group, and the remaining capsules were calculated and reported on the data collection sheet. The subjects were also asked to report the general expected side effects due to any intervention, both positive and negative records, after the intervention period at week 10 .

\section{Data collection}

Data collection was carried out at preassigned community halls known to all subjects. All subjects were informed on phone to fast on the assessment day. The baseline assessment included a detailed sociodemographic data, medical history, frailty status assessment, physical function, cognitive function, nutritional status, and selected frailty biomarkers. The second assessment occurred at week 5 , which included frailty status assessment, physical function, cognitive function, and nutritional status. The last assessment occurred at week 10 and included the same assessments as per baseline. All the assessments were carried out by trained postgraduates team in health sciences (from three different disciplines: nutrition, physiotherapy, and biomedical sciences) who were completely blinded about the intervention groups.

\section{Study instruments and tools}

\section{Frailty assessment}

Fried criteria, a well-known frailty assessment tool that is widely used in clinical research, ${ }^{28}$ were utilized in this study. The criteria included assessment of shrinking, exhaustion, weakness, slowness, and low physical activity. Till date, there are no Malaysian cutoff points for hand grip and walking speed, and so the original cutoff points from Fried et al were used. ${ }^{27}$ The second tool was the Frailty Index (FI) accumulation of deficit (40 items), which is the short form of the original 70-item FI accumulation of deficits. This tool is reported to be reliable for frailty assessment in clinical research. ${ }^{28}$ It is recommended to be used in intervention studies for frailty prevention and treatment. ${ }^{29}$ In the present study, FI assessment was done following the standard procedures described and standardized in a study by Searle et al. ${ }^{30}$ The convergent validity of the two frailty assessment instruments was tested by correlating the measures with each other and other health status measures. ${ }^{28}$ Significant correlations were found and the two instruments were recommended to be used in frailty assessment in clinical research. Physical activity was assessed using Physical Activity Scale for Elderly (PASE). ${ }^{31}$ In a recent study, PASE was also validated in Asian population, with acceptable test-retest reliability (intraclass correlation coefficient $=0.81) .{ }^{32}$

\section{Nutritional status assessment}

Nutritional status assessment included anthropometric measurements (weight, height, mid-upper arm circumferences, waist circumferences, hip circumferences, and calf circumferences) and was conducted by trained postgraduates in health sciences according to standard techniques. ${ }^{33}$ Height measurement was done using a height measuring device (Seca 214), to the nearest $0.1 \mathrm{~m}$. Body weight was measured using a digital scale (Tanita HD-319 scale, Sin Huat Hin Machinery (M) Sdn.Bhd., Serdang, Kuala Lumpur, Malaysia), to the nearest $0.1 \mathrm{~kg}$. Circumferences measurements were done using a flexible, nonextensible measuring tape. Body composition was done using bio electric impedance (BIA) (Korean brand, model Inbody S10 ${ }^{\circledR}$, Inbody Malaysia, Kuala Lumpur, Malaysia). Fat mass, fat percentage, fat free mass, soft lean mass, skeletal muscle mass, and basal metabolic rate were extracted from the machine output. Cognitive function was assessed through the MMSE, ${ }^{34}$ using the validated Malaysian version. ${ }^{35}$ Physical function assessment included hand grip test, 30-second chair stand test, 8-ft time up and go test, 2-minute step test, gait speed test at rapid pace, peak expiratory flow rate (PEFR) test, and shoulder strength test. The subjects were given demonstration on each test before they performed the tests. The hand grip test was done using a digital hand dynamometer (Jamar Plus, Model: 563213, USA), among the community dwelling elderly; the reliability of grip strength using the dynamometer was 0.954 using test and retest reliability. ${ }^{36}$ Shoulder strength test was done using customized set, consisting of digital gauge and adjustable Velcro loop with interlock straps to adjust it according to the subject's height. The reliability test of the shoulder strength was done using test and retest method and the correlation 
coefficient ranged from 0.96 to $0.98 .{ }^{37}$ The 30 -second chair stand test was performed to assess the lower limbs strength; this test was reported to be a valid and reliable test for measuring lower body strength among the community dwelling elderly. ${ }^{38}$ The 8 -ft time up and go test was used to assess mobility and balance. This test is one of the basic functional and mobility tests for the elderly with a documented validity and reliability study. ${ }^{39} \mathrm{With}$ respect to gait speed test, different distances all have excellent reliability range from 0.96 to 0.99 ; also, $6 \mathrm{~m}$ gait speed was validated by concurrent validity of 4 and $10 \mathrm{~m}$ walling test. ${ }^{40}$ The 2 -minute step test was included in the test battery as an aerobic endurance assessment. The PEFR test was done for respiratory function assessment; it was done using peak flow meter (Mini-Wright, USA) with a disposable mouth piece. All of the fore mentioned physical function tests were done following the standard procedures in senior fitness test. Subjects were asked to perform the tests twice with 5 minutes rest between the two trials. The activity of daily living was assessed using Katz Index of Independent in Activities of Daily Living scale. ${ }^{41}$ The instrumental activity of daily living was assessed using Lawton Instrumental Activities of Daily Living scale (IADL).

A total amount of $20 \mathrm{~mL}$ fasting venous blood was drawn at baseline and week 10 to assess the biochemical parameters. The blood samples were immediately transferred from the place of data collection to the Pathology and Clinical Laboratory (M) Sdn Bhd. The samples were centrifuged using TOMY Flexin Bench Top centrifuge at $350 \mathrm{rpm} / 5$ minutes, aliquoted in micro centrifuge tubes, and then stored at $-80^{\circ} \mathrm{C}$. The biochemical analysis for the routine tests, including fasting blood sugar, $\mathrm{HBA}_{1 \mathrm{c}}$, lipid profile, kidney function, and liver function, was done at the lab.

Frailty biomarkers, including interleukin (IL)-6, tumor necrosis factor (TNF)- $\alpha$, and insulin-like growth factor (IGF)-1, in addition to plasma free L-carnitine and total L-carnitine were analyzed at the Bioserasi Laboratory of Universiti Kebangsaan Malaysia. The serum level of L-carnitine and total L-carnitine was done following a Prieto et al method. ${ }^{42}$ Detection of IL-6, TNF- $\alpha$, IGF-1, and vitamin $\mathrm{D}$ in the serum was done using enzyme-linked immunosorbent assay kits (R\&D Systems, Inc., Minneapolis, MN, USA) and assessed using spectrophotometry.

\section{Statistical analysis}

All statistical analyses were carried out using the Statistical Package for Social Sciences software, version 21.0 (IBM Corporation, Armonk, NY, USA). An alpha level of 0.05 was considered for all the statistical tests used in the study. Normality test was checked for all of the continuous variables and the data were not normally distributed. Mann-Whitney test was employed for the continuous variables and chi-square for the categorical variables to determine the characteristics differences between the two groups (ie, L-carnitine and placebo). The effects of the intervention on the outcome variables for each group were assessed using the nonparametric tests (Friedman test and Wilcoxon test). Also, the percentage of mean change was calculated for each subject from baseline to week 5 and from baseline to week 10, and then Mann-Whitney test was employed to determine the difference in the mean of the percentage of mean difference between the two groups.

\section{Results Subjects characteristics}

A total of 50 subjects out of 58 were recruited and completed the study, with a response rate of $89.7 \%$. Only six subjects dropped out and two subjects were excluded from the final analysis due to acute disease on the day of the data collections. Both groups had an almost similar age mean (68.2 \pm 6.3 years for the intervention group and $68.8 \pm 6.5$ years for the placebo group). There were no significant differences between the two groups in terms of sex, ethnicity, marital status, living arrangement, level of education, and employment status and also medical history (with an exception of vision problem), as shown in Table 1 . There were also no significant differences between groups on frailty status score,

Table I Sociodemographic characteristics of the L-carnitine and placebo groups

\begin{tabular}{|c|c|c|c|}
\hline Parameter & $\begin{array}{l}\text { L-carnitine } \\
\text { group }(n=26)\end{array}$ & $\begin{array}{l}\text { Placebo } \\
\text { group }(n=24)\end{array}$ & $P$-value \\
\hline Mean age (years) & $68.2 \pm 6.3$ & $68.8 \pm 6.5$ & $0.742^{\mathrm{a}}$ \\
\hline \multicolumn{4}{|l|}{ Sex } \\
\hline Male & $14(53.8 \%)$ & $9(37.5 \%)$ & $0.191^{b}$ \\
\hline Female & 12 (46.2\%) & 15 (62.5\%) & \\
\hline \multicolumn{4}{|l|}{ Race } \\
\hline Malay & 20 (76.9\%) & 19 (79.2\%) & $0.656^{b}$ \\
\hline Chinese & $2(7.7 \%)$ & $2(8.3 \%)$ & \\
\hline Indian & $4(15.4 \%)$ & $3(8.3 \%)$ & \\
\hline \multicolumn{4}{|l|}{ Marital status } \\
\hline Married & $21(80.8 \%)$ & 17 (70.8\%) & $0.313^{b}$ \\
\hline Not married & 5 (19.2\%) & 7 (29.2\%) & \\
\hline \multicolumn{4}{|l|}{ Living status } \\
\hline Living alone & $2(7.7 \%)$ & $\mathrm{I}(4.2 \%)$ & $0.534^{b}$ \\
\hline Living with company & $24(92.3 \%)$ & $23(95.8 \%)$ & \\
\hline \multicolumn{4}{|l|}{ Level of education } \\
\hline $\begin{array}{l}\text { Mean schooling } \\
\text { period (years) }\end{array}$ & $10 \pm 3$ & $10 \pm 3$ & $\mathrm{I}^{\mathrm{a}}$ \\
\hline \multicolumn{4}{|l|}{ Employment status } \\
\hline Working & $3(12 \%)$ & I (4.2\%) & $0.324^{b}$ \\
\hline Not working & 22 (88\%) & 23 (95.8\%) & \\
\hline
\end{tabular}

Notes: aMann-Whitney test, ${ }^{\mathrm{b}}$ chi-square test. 
physical function, nutritional status, and the biochemical tests using Mann-Whitney test.

Friedman test was employed to determine the changes in the mean of the outcome variables at baseline, week 5, and week 10. Results showed that there was a significant improvement in the mean FI score and hand grip test in the L-carnitine group $(P<0.05$ for both parameters) while there were no significant changes in the mean scores in the placebo group. The changes in the mean of the functional tests (activity of daily living [ADL], instrumental activity of daily living [IADl], and physical function) were not significant in both L-carnitine and placebo group, except for shoulder strength test and physical activity (PASE) of which both groups showed significant improvements $(P<0.05$ for all parameters). The nonparametric Wilcoxon test was employed to determine the changes in the frailty biomarkers and L-carnitine derivatives before and after the intervention. The results showed no significant differences in the blood level of these biomarkers after the intervention. A favorable effect was noticed on the level of IL-6 and IGF-1, but this effect did not reach a significant level. The changes in the free L-carnitine, total L-carnitine, and acyl L-carnitine were not significant in both groups, as shown in Table 2. There were no significant changes in the secondary outcome variables: nutritional status, body composition, and cognitive using Friedman test. Similarly, there were no significant changes in fasting blood sugar, $\mathrm{HBA}_{1 \mathrm{c}}$, lipid profile, liver function, and kidney function using Wilcoxon test, for all of the tests.

Further analysis was done by calculating the percentage of mean changes from the outcome variables at 5 and 10 weeks. A significant favorable effect of L-carnitine was recorded in frailty score and hand grip $(P<0.01, P<0.05)$, respectively, as shown in Figure 2. The percent change in FI score from baseline to 10 weeks was $-34.4 \%$ in L-carnitine group compared to $-2.1 \%$ in placebo group $(P<0.001)$ (Figure $2 \mathrm{~A})$. The corresponding figures for handgrip strength was $7.2 \%$ for the L-carnitine group as compared to $-3.6 \%$ in the placebo group $(P<0.05)$ (Figure 2B). There were favorable effects and improvements in some of other physical function tests, but these improvements were not significant between the groups, such as the improvement in PEFR test (Figure 2C).

Figure 3 shows the percent change in all of the biomarkers tests from baseline to 10 weeks. Significant differences between the groups were not found for all of the tested biomarkers. However, it is clearly noticeable that the decrease in percent change for IL-6 level was better in the L-carnitine group compared to placebo group. A favorable increase in the IGF-1 level in the L-carnitine group was also observed compared to placebo group. The rest of the outcome variables,
Table 2 Effects of the intervention on the primary outcome variables for the L-carnitine and placebo groups (presented as mean \pm SD)

\begin{tabular}{|c|c|c|c|c|}
\hline Parameters & $\begin{array}{l}\text { L-carnitine } \\
\text { group } \\
\text { (mean } \pm \text { SD) }\end{array}$ & $P$-value & $\begin{array}{l}\text { Placebo } \\
\text { group } \\
\text { (mean } \pm \text { SD) }\end{array}$ & $P$-value \\
\hline \multicolumn{5}{|c|}{ Frailty Index score } \\
\hline Baseline & $0.112 \pm 0.065$ & $0.00 \mathrm{I}^{\mathrm{a}}$ & $0.118 \pm 0.045$ & $0.764^{\mathrm{a}}$ \\
\hline 5 weeks & $0.097 \pm 0.063$ & & $0.116 \pm 0.056$ & \\
\hline 10 weeks & $0.076 \pm 0.0578$ & & $0.111 \pm 0.062$ & \\
\hline \multicolumn{5}{|l|}{ Hand grip (kg) } \\
\hline Baseline & $23.6 \pm 6.9$ & $0.012^{\mathrm{a}}$ & $22.8 \pm 6.5$ & $0.058^{\mathrm{a}}$ \\
\hline 5 weeks & $23.6 \pm 7.3$ & & $21.2 \pm 6.7$ & \\
\hline 10 weeks & $24.4 \pm 7.4$ & & $21.7 \pm 5.4$ & \\
\hline \multicolumn{5}{|c|}{ 2-minute step test (step) } \\
\hline Baseline & $69 \pm 22$ & $0.350^{\mathrm{a}}$ & $93 \pm 31$ & $0.828^{a}$ \\
\hline 5 weeks & $72 \pm 27$ & & $83 \pm 32$ & \\
\hline 10 weeks & $76 \pm 23$ & & $85 \pm 23$ & \\
\hline \multicolumn{5}{|c|}{ Time up and go (s) } \\
\hline Baseline & $12.2 \pm 8.5$ & $0.23 \mathrm{I}^{\mathrm{a}}$ & $9.5 \pm 2.6$ & $0.097^{\mathrm{a}}$ \\
\hline 5 weeks & $9.2 \pm 2.9$ & & $8.9 \pm 3.1$ & \\
\hline 10 weeks & $9.4 \pm 1.9$ & & $9.5 \pm 2.9$ & \\
\hline \multicolumn{5}{|l|}{ Usual pace (s) } \\
\hline Baseline & $6.5 \pm 1.2$ & $0.234^{\mathrm{a}}$ & $6.6 \pm 1.6$ & $0.106^{\mathrm{a}}$ \\
\hline 5 weeks & $6.2 \pm 1.5$ & & $6.5 \pm 1.4$ & \\
\hline 10 weeks & $6.4 \pm 1.0$ & & $6.9 \pm 1.6$ & \\
\hline \multicolumn{5}{|l|}{ Rapid pace (s) } \\
\hline Baseline & $4.9 \pm 0.7$ & $0.509^{a}$ & $5.1 \pm 1.3$ & $0.397^{a}$ \\
\hline 5 weeks & $5.1 \pm 1.3$ & & $5.0 \pm 1.5$ & \\
\hline 10 weeks & $4.9 \pm 0.9$ & & $5.2 \pm 1.3$ & \\
\hline \multicolumn{5}{|c|}{ Shoulder strength (kg) } \\
\hline Baseline & $11.7 \pm 5.7$ & $0.022^{\mathrm{a}}$ & $11.3 \pm 5.2$ & $0.009 * *, a$ \\
\hline 5 weeks & $11.9 \pm 5.6$ & & $11.4 \pm 4.9$ & \\
\hline 10 weeks & $13.8 \pm 5.9$ & & $13.5 \pm 4.7$ & \\
\hline \multicolumn{5}{|c|}{ Peak expiratory flow rate (L/min) } \\
\hline Baseline & $400 \pm 100$ & $0.24 I^{\mathrm{a}}$ & $380 \pm 100$ & $0.675^{\mathrm{a}}$ \\
\hline 5 weeks & $415 \pm 90$ & & $390 \pm 80$ & \\
\hline 10 weeks & $410 \pm 80$ & & $380 \pm 90$ & \\
\hline \multicolumn{5}{|c|}{ Chair stand test (times/30 s) } \\
\hline Baseline & $11.0 \pm 3.3$ & $0.07^{a}$ & $11.3 \pm 3.6$ & $0.05^{\mathrm{a}}$ \\
\hline 5 weeks & $11.5 \pm 3.8$ & & $12.1 \pm 3.69$ & \\
\hline 10 weeks & $11.3 \pm 3.8$ & & $11.1 \pm 4$ & \\
\hline \multicolumn{5}{|c|}{ Physical activity scale PASE } \\
\hline Baseline & $155 \pm 60$ & $0.025^{\mathrm{a}}$ & $150 \pm 70$ & $0.013^{*, a}$ \\
\hline 5 weeks & $120 \pm 40$ & & $110 \pm 40$ & \\
\hline 10 weeks & $150 \pm 50$ & & $130 \pm 30$ & \\
\hline \multicolumn{5}{|c|}{ Activity of daily living } \\
\hline Baseline & $6 \pm 0$ & NA & $6 \pm 0$ & NA \\
\hline 5 weeks & $6 \pm 0$ & & $6 \pm 0$ & \\
\hline 10 weeks & $6 \pm 0$ & & $6 \pm 0$ & \\
\hline \multicolumn{5}{|c|}{ Instrumental activity of daily living } \\
\hline Baseline & $13.9 \pm 0.4$ & $0.765^{\mathrm{a}}$ & $13.6 \pm 0.9$ & NA \\
\hline 5 weeks & $13.9 \pm 0.4$ & & $13.6 \pm 0.9$ & \\
\hline 10 weeks & $13.9 \pm 0.5$ & & $13.6 \pm 0.9$ & \\
\hline \multicolumn{5}{|c|}{ Frailty biomarkers } \\
\hline \multicolumn{5}{|c|}{ Interleukin-6 (pg/mL) } \\
\hline Baseline & $6.1 \pm 2.9$ & $0.699^{b}$ & $6.2 \pm 2.3$ & $0.05^{b}$ \\
\hline 10 weeks & $5.9 \pm 7.3$ & & $8.9 \pm 8.2$ & \\
\hline
\end{tabular}


Table 2 (Continued)

\begin{tabular}{|c|c|c|c|c|}
\hline Parameters & $\begin{array}{l}\text { L-carnitine } \\
\text { group } \\
\text { (mean } \pm \text { SD) }\end{array}$ & $P$-value & $\begin{array}{l}\text { Placebo } \\
\text { group } \\
(\text { mean } \pm \text { SD) }\end{array}$ & $P$-value \\
\hline \multicolumn{5}{|c|}{ Tumor necrosis factor- $\alpha(\mathrm{pg} / \mathrm{mL})$} \\
\hline Baseline & $5.9 \pm 2.0$ & $0.802^{\mathrm{b}}$ & $6.2 \pm 2.1$ & $0.670^{\mathrm{b}}$ \\
\hline 10 weeks & $5.9 \pm 4.9$ & & $5.8 \pm 3.9$ & \\
\hline \multicolumn{5}{|c|}{ Insulin-like growth factor-I (ng/mL) } \\
\hline Baseline & $92.7 \pm 37.8$ & $0.585^{\mathrm{b}}$ & $102.2 \pm 47.5$ & $0.898^{b}$ \\
\hline 10 weeks & $103.9 \pm 65$ & & $99.7 \pm 65.3$ & \\
\hline \multicolumn{5}{|c|}{ Total L-carnitine $(\mu \mathrm{mol} / \mathrm{L})$} \\
\hline Baseline & $36.1 \pm 14.3$ & $0.606^{\mathrm{b}}$ & $35.6 \pm 14.8$ & $0.130^{\mathrm{b}}$ \\
\hline 10 weeks & $38.8 \pm 18.1$ & & $37 \pm 20.6$ & \\
\hline \multicolumn{5}{|c|}{ Free L-carnitine $(\mu \mathrm{mol} / \mathrm{L})$} \\
\hline Baseline & $32.4 \pm 12.4$ & $0.845^{b}$ & $26.9 \pm 11.6$ & $0.516^{b}$ \\
\hline 10 weeks & $33.9 \pm 5.1$ & & $31.1 \pm 3.3$ & \\
\hline \multicolumn{5}{|c|}{ Acyl L-carnitine $(\mu \mathrm{mol} / \mathrm{L})$} \\
\hline Baseline & $3.6 \pm 14.9$ & $0.905^{\mathrm{b}}$ & $8.6 \pm 15.2$ & $0.819^{b}$ \\
\hline 10 weeks & $3.1 \pm 20.4$ & & $7.6 \pm 17.8$ & \\
\hline
\end{tabular}

Notes: $* P<0.05, * * P<0.01$, ausing Friedman test, ${ }^{b}$ using Wilcoxon test. Abbreviations: PASE, Physical Activity Scale for Elderly; SD, standard deviation; NA, not applicable.

nutritional status, body composition, biochemical data, and cognitive function, did not show any significant differences between the L-carnitine and placebo groups in the percent of mean changes in the intervention period.

\section{The transition in the frailty status after the intervention}

Figure 4A shows the changes in the frailty scores for the L-carnitine group. Three subjects (11.5\%) changed from prefail to normal. The percentage of subjects with one deficit increased from 18 subjects $(69.2 \%)$ to 19 subjects (73.1\%) after 10 weeks, while the percentage of subjects with two deficits decreased from ten subjects (30.8\%) to four subjects (15.4\%) after 10 weeks' intervention. Figure 4B shows the changes in frailty scores in the placebo group. The improvement in the frailty status was less compared to L-carnitine group. Only one subject (4.2\%) improved to normal status. No changes in the percentage of subjects with one deficit were observed, while the percentage decreased from six subjects $(25 \%)$ to five subjects $(20.8 \%)$ for those with two deficits. Figure 5 shows that four subjects (15.1\%) with weakness were improved in L-carnitine group, while no improvement was found in the placebo group. For the exhaustion criteria, four subjects (15.4\%) with exhaustion in the L-carnitine group changed to normal, while only one subject $(4.2 \%)$ in the placebo group changed to normal in exhaustion criteria. For the low physical activity criteria, there were no changes in the L-carnitine group, while in the placebo group one subject (4.1\%) changed from being mild to a moderately active status in terms of physical activity.

With respect to side effect recorded following the supplementation, it was found that there was a significantly higher percentage of subjects in the L-carnitine group. They reported feeling more energetic after the intervention $(42.3 \%)$ as compared to the placebo group $(16.7 \%)(P<0.05)$. No significant adverse effects were reported in both the placebo and intervention groups (Table 3 ).

\section{Discussion}

In the present study, both male and female subjects from three races composing of the Malaysian community were included. This made the sample representative of the study population (prefrail community dwelling Malaysian older adults). The dropout rate was $10.3 \%$. Only six subjects dropped out, and two subjects were excluded from the final
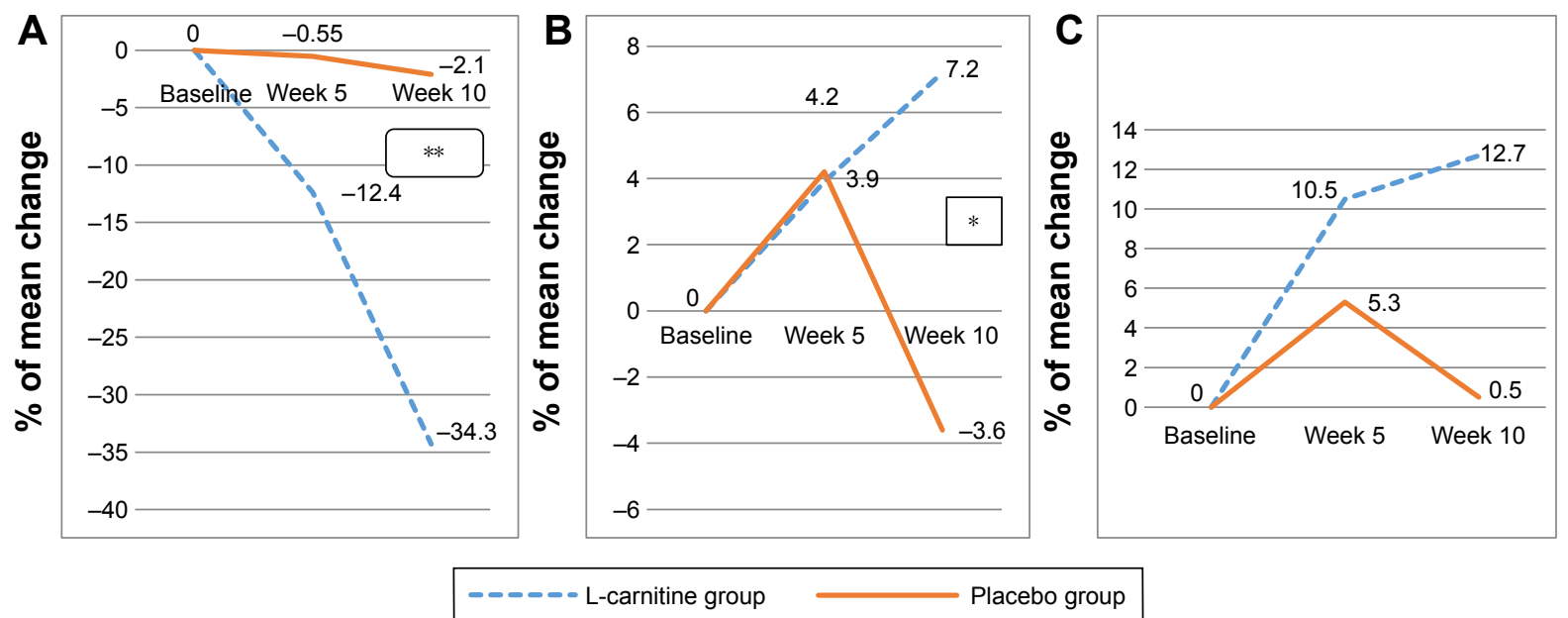

Figure 2 Percent change in Frailty Index score and physical function.

Notes: (A) Frailty Index, (B) Hand grip, (C) PEFR $* P<0.05$, $* * P<0.01$, using independent $t$-test/Mann-Whitney test.

Abbreviation: PEFR, peak expiratory flow rate. 

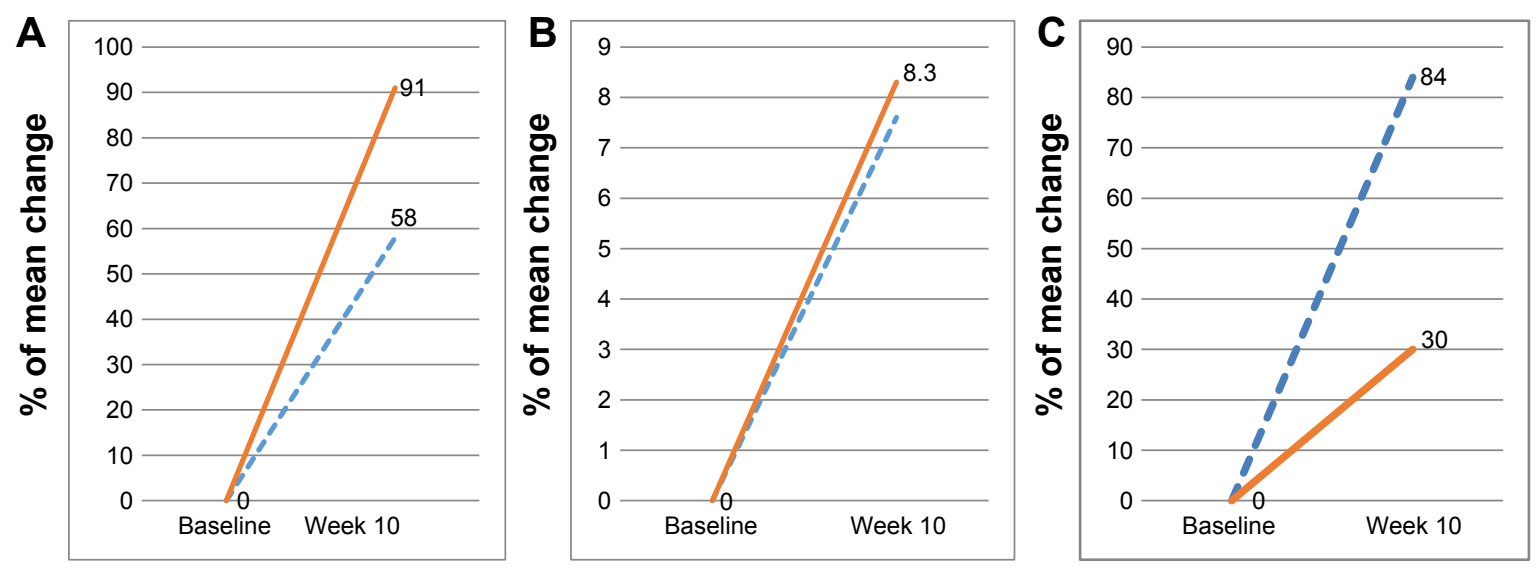

Placebo group

Figure 3 Percent of mean change in frailty in selected biomarkers.

Notes: (A) IL-6, (B) TNF- $\alpha$, (C) IGF-I, no significant differences were found using independent $t$-test/Mann-Whitney test.

Abbreviations: IL-6, interleukin 6; IGF-I, insulin-like growth factor-I; TNF- $\alpha$, tumor necrosis factor-alpha.
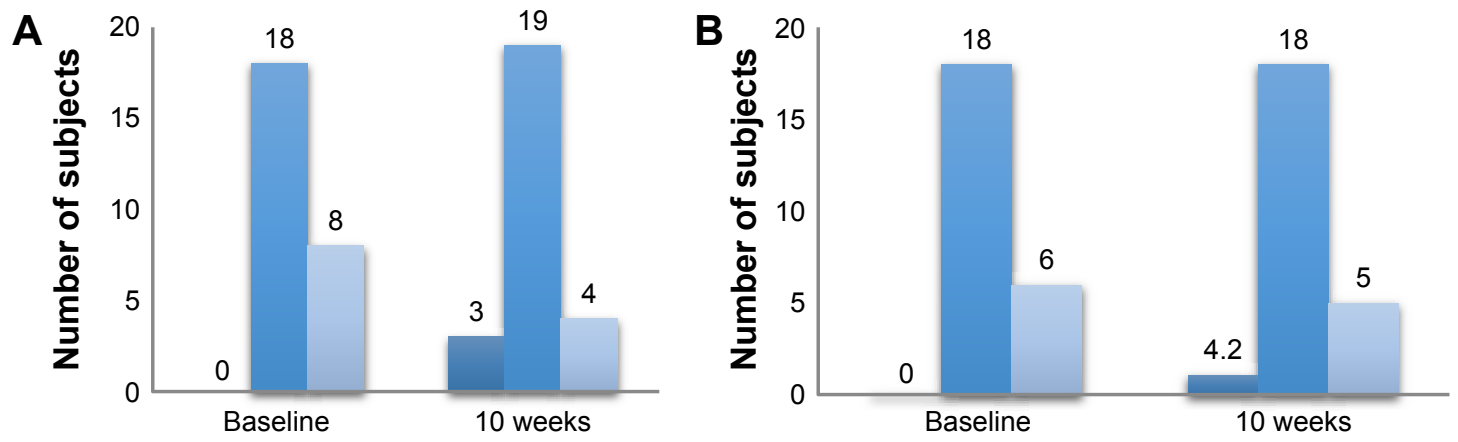

0 defict 1 defict 2 deficit

Figure 4 Changes in the frailty scores according to Fried criteria.

Notes: (A) L-carnitine group, (B) Placebo group.
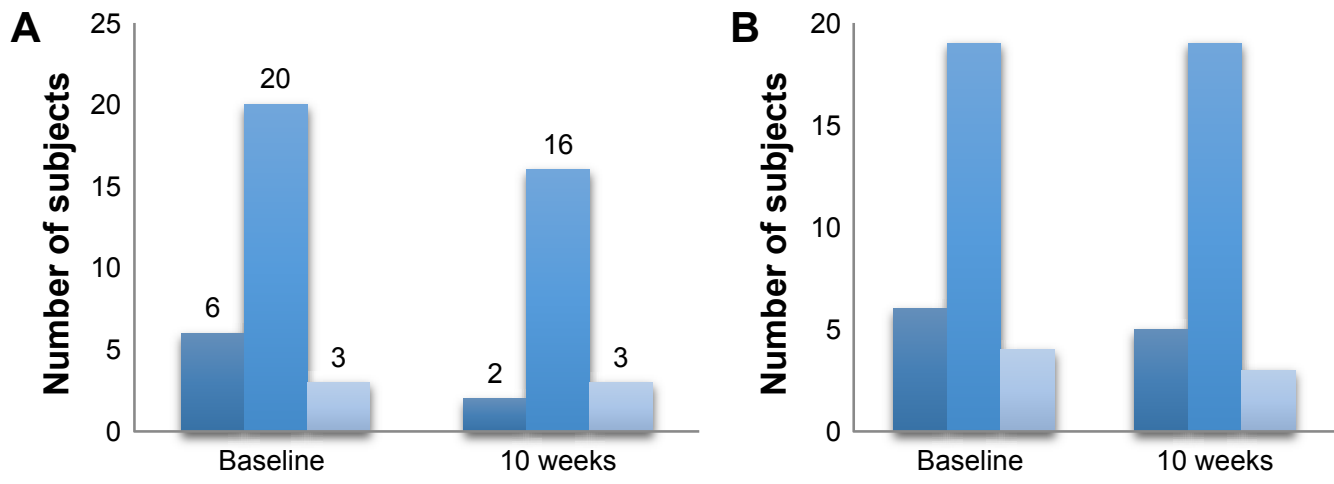

Exhaustion Weakness Low physical activity

Figure 5 Changes in the frailty deficit percentage before and after the intervention.

Notes: (A) L-carnitine group, (B) placebo group. 
Table 3 Self-reported side effect by subjects (presented as n [\%])

\begin{tabular}{|c|c|c|c|}
\hline Side effect & $\begin{array}{l}\text { L-carnitine } \\
\text { group (\%) }\end{array}$ & $\begin{array}{l}\text { Placebo } \\
\text { group (\%) }\end{array}$ & $P$-value \\
\hline \multicolumn{4}{|c|}{ General feeling } \\
\hline Normal & $19(73.1)$ & $17(70.8)$ & 0.554 \\
\hline Better & $7(26.9)$ & $7(29.2)$ & \\
\hline Worse & $0(0)$ & $0(0)$ & \\
\hline \multicolumn{4}{|c|}{ Feeling more energetic } \\
\hline Yes & II (42.3) & $4(16.7)$ & $0.047^{*}$ \\
\hline No & $15(57.7)$ & $20(83.3)$ & \\
\hline \multicolumn{4}{|l|}{ Nausea } \\
\hline Yes & $0(0)$ & $0(0)$ & 0.674 \\
\hline No & $26(100)$ & $24(100)$ & \\
\hline \multicolumn{4}{|l|}{ Vomiting } \\
\hline Yes & $0(0)$ & $0(0)$ & 0.603 \\
\hline No & $26(100)$ & $24(100)$ & \\
\hline \multicolumn{4}{|l|}{ Diarrhea } \\
\hline Yes & $0(0)$ & $0(0)$ & 0.456 \\
\hline No & $26(100)$ & $24(100)$ & \\
\hline \multicolumn{4}{|l|}{ Constipation } \\
\hline Yes & I (3.8) & $0(0)$ & 0.476 \\
\hline No & $25(96.2)$ & $24(100)$ & \\
\hline \multicolumn{4}{|l|}{ Muscle pain } \\
\hline Yes & $0(0)$ & I (4.2) & 0.334 \\
\hline No & $26(100)$ & $23(95.8)$ & \\
\hline \multicolumn{4}{|l|}{ Appetite } \\
\hline Increased & $2(7.7)$ & $0(0)$ & 0.443 \\
\hline Same & $23(88.5)$ & $20(83.3)$ & \\
\hline Decrease & I (3.8) & $4(16.7)$ & \\
\hline \multicolumn{4}{|l|}{ Sleep } \\
\hline More & $0(0)$ & $\mathrm{I}(4.2)$ & 0.443 \\
\hline Same & $26(100)$ & $23(95.8)$ & \\
\hline Less & $0(0)$ & $0(0)$ & \\
\hline
\end{tabular}

Note: $* P<0.05$ using chi-square test.

analysis. The intervention of the L-carnitine supplementation was well tolerated with good compliance; no adverse events or symptoms were reported from the subjects in both groups (placebo and L-carnitine).

To the best of our knowledge, this study is the first study of its kind that was conducted to investigate the effects of the L-carnitine supplement on frailty status either among the frail or prefrail elderly. Significant improvement in the L-carnitine group was only reported in the FI score and hand grip test. Moreover, the percentage of mean changes scores were significantly different between the two groups in frailty status and hand grip test. There were also no significant changes in the slowness criteria measure using gait speed between the L-carnitine and placebo groups. This might have happened due to the very small number (three) of the subjects who were found to be slow during baseline. Thus, the effects of L-carnitine in improving gait speed were not found. In this study, it appears that L-carnitine has a significant effect in improving frailty status in general, and hand grip test and exhaustion in particular. This improvement can be explained by the possible effect of L-carnitine in enhancing the mitochondrial function. This effect on mitochondria function includes correcting the cellular energy supply deficit, through facilitating the transport of long chain fatty acid - the main source of energy in skeletal muscle - into the mitochondria; facilitating the removal of short and medium chain fatty acid that accumulate during metabolism; and maintaining the proteins and lipids in the optimum level. ${ }^{43}$ L-carnitine supplementation may increase the rate of fatty acid oxidation, preserve muscle glycogen, and increase the production of the adenosine triphosphate (ATP). ${ }^{21}$ These suggested mechanisms improve the energetic metabolism in muscular skeletal tissue, and may have improved muscle function and reduced fatigue and exhaustion.

The findings of this study regarding the effect of L-carnitine on frailty were compared with other studies that reported the effects of L-carnitine supplementation among older adults. However, it should be noted that the findings were different and varied depending on the subjects' inclusion criteria and the targeted outcomes. In the present study, the effect of L-carnitine on frailty status was assessed using the FI accumulation of deficit tool. The results showed that FI scores significantly improved. Previous studies did not use the FI tool to define frailty status changes with L-carnitine supplementation; therefore, the effect of L-carnitine on frailty was further discussed according to its effects on Fried criteria (2001) and other physical and functional status tests. The effect of the intervention on hand grip test was significant, evidenced by significant differences in the percent of mean changes between the two intervention arms. It is well known that skeletal muscle is L-carnitine-dependent, as this muscle depends on fatty acid oxidation as the main source of energy. ${ }^{44}$

Exhaustion criteria improved due to the L-carnitine intervention evidenced by the significant association found between the L-carnitine group and self-report feelings of being more energetic. This finding is in line with other previous studies conducted on different groups of older adults and reported significant improvement in the physical and mental fatigue with the L-carnitine supplementation in different intervention periods, different intervention doses, and different L-carnitine derivatives..$^{21,22,45}$

With respect to the criteria of low physical activity, the intervention with either L-carnitine or placebo showed significant changes in the PASE score using Friedman tests, but the percent of mean changes did not show significant 
difference between the two groups using Mann-Whitney test. Literature regarding intervention studies to determine the effects of L-carnitine on the physical activity among older adults or other subject groups is limited.

The effects of L-carnitine on functional status (ADL and IADL) and physical function were examined. Changes in the subjects' functional status (ADL and IADL) were not observed, implying that the effects on ADL and IADL were not significant. This could be attributed to the fact that that all subjects in both intervention arms were completely independent at baseline, as indicated by the full scores of ADL and very high scores of IADL.

The effects of L-carnitine on physical function was assessed by determining these effects on the eight physical function tests, including hand grip test, walking speed test, 2-minute step test, time up and go test, chair stand test, rapid pace test, shoulder strength test, and PEFR test. Despite the nonsignificant effects found from all these tests, the trend of the improvement was reported for the 2-minute step test, time up and go test, shoulder strength test, and PEFR test. In other words, the mean changes in these tests were toward the improvement in L-carnitine group compared to placebo. This might be due to the intervention period and dosage of L-carnitine.

The results of this study revealed no significant changes in the frailty biomarkers: IL-6, TNF- $\alpha$, and IGF-1. However, the effects on IL-6 and IGF-1 were favorable. The majority of nutrition intervention studies targeted frailty as a major outcome; they assessed the effectiveness of the frailty using physical function, body composition and physical and cognitive function, with less focus on frailty biomarkers. However, few studies targeted the frailty biomarkers were found. L-carnitine theoretically had the ability to modulate the inflammatory status..$^{9,46}$

In clinical studies, L-carnitine showed a significant effect on modulating the inflammatory mediators C-reactive proteins $^{47,48}$ and IL- 6 in hemodialysis patients. ${ }^{49}$ A high dose of L-carnitine ( $6 \mathrm{~g} /$ day) has a significant reduction effect on the TNF- $\alpha$ level in HIV subjects. ${ }^{18}$ From these reviewed studies, it can be concluded that L-carnitine modulates the hyperinflammation. Even in this documented effect, the mechanisms of how L-carnitine regulates this inflammation are not well understood. It was suggested that the reduction of the C-reactive protein and TNF- $\alpha$ levels is due to the effect of L-carnitine on reducing the level of IL-6, but the mechanism of how L-carnitine reduces IL-6 is still unknown. ${ }^{49}$ But this study did not find this effect, which may be due to the fact that the subjects were prefrail and the inflammatory mediator level was not high, such as in the hemodialysis and HIV patients, and it may be due to the doses and intervention periods.

The results of the study revealed that the changes in free L-carnitine, total L-carnitines, and acyl L-carnitine blood level were not significant between the two groups. This means that the intervention with L-carnitine had no effect on the blood level of free, total, and acyl L-carnitine. Previous intervention studies of L-carnitine among the elderly were reviewed. The intervention with $2 \mathrm{~g}$ /day of L-carnitine for 6 months among centenarians showed no significant changes on free and total L-carnitine blood levels. ${ }^{50}$ The consistent findings may be due to the fact that L-carnitine is a nonessential amino acid, which the body can synthesize from other amino acids in presence of other micronutrients. ${ }^{50}$ Other reasons are the differences of the rate of L-carnitine uptake from different tissues, and the variation of storage of L-carnitine among different tissues (hepatic, renal, cardiac, skeletal muscle, and brain). ${ }^{21}$ Cavallini et $\mathrm{al}^{45}$ and Malaguarnera et $\mathrm{al}^{21}$ conducted intervention studies among the elderly, but they did not report the L-carnitine blood level in their published data.

The current study did not find any significant effect on cognitive function among the study subjects. However, a previous study that included 66 centenarian adults who received L-carnitine supplementation showed significant improvement in MMSE due to the intervention. ${ }^{21}$ Subjects' characteristics in the two studies might play a role in these differences. For instance, the participants' mean score of MMSE was very low (16.5) and the subjects had cognitive impairment, ${ }^{21}$ whereas in this study, the mean score of MMSE, which was very high (27), indicated a normal cognitive function. So, it is not enough to conclude that L-carnitine has no effect on cognitive function based on the findings of the current study. Further research needs to be conducted on mild cognitive impairment subjects to support the findings reported in the literature.

\section{Conclusion}

In conclusion, L-carnitine supplementation has the potential to improve physical function and reverse frailty, particularly for those suffering from fatigue and weakness with a convenient dose. Further research is needed to determine the efficacy of the L-carnitine supplementation among older adults with different frailty status, that is, frail, for a longer intervention period, different doses, and also combined with exercise.

This study utilized a randomized, placebo-controlled, double-blind, multicenter study design, a full 10-week supplementation, blood monitoring at baseline and 
posttreatment, and multiple assessments of various functions. However, this study is not without its limitations. It assessed the efficacy of the L-carnitine supplementation in short-term (10 weeks) and did not assess the efficacy for a longer period. Fatigue assessment was done using subjective methods. The nonstatistical significances in this study were assigned due to the low dose of L-carnitine given and the subjects' frailty status. The study did not measure the L-carnitine excretion from urine before and after the intervention, which might provide further explanation of L-carnitine distribution inside the human body. In the screening phase, the main limitations occurred in the frailty assessment tools. For Fried criteria, the study used the cutoff points of hand grip and gait speed from the original study published by Fried et $\mathrm{al}^{27}$ conducted on a Western population rather than Asian.

\section{Acknowledgments}

We would like to acknowledge the financial support from the Ministry of Education in the Long Term Research Grant Scheme (LRGS/BU/2012/UKM-UKM/K/01) and also the direct and indirect input from the research team toward the study. We would like to extend special acknowledgments to the Malaysian older adults who agreed to participate in the study, cooperated with us during the data collection and assessment, and showed good compliance and interest to finish the study.

\section{Disclosure}

The authors report no conflicts of interest in this work.

\section{References}

1. Hung CW, Chen YC, Hsieh WL, Chiou SH, Kao CL. Ageing and neurodegenerative diseases. Ageing Res Rev. 2010;9(Suppl 1):S36-S46.

2. Sourdet S, Rougé-Bugat M, Vellas B, Forette F. Frailty and aging. J Nutr Health Aging. 2012;16(4):283-284.

3. Bieniek J, Wilczyński K, Szewieczek J. Fried frailty phenotype assessment components as applied to geriatric inpatients. Clin Interv Aging. 2016;11:453-459.

4. Clegg A, Young J. The frailty syndrome. Clin Med. 2011;11(1):72-75.

5. Weiss CO. Frailty and chronic diseases in older adults. Clin Geriatr Med. 2011;27(1):39-52.

6. Clegg A, Young J, Iliffe S, Rikkert MO, Rockwood K. Frailty in elderly people. Lancet. 2013;381(9868):752-762.

7. Chen X, Mao G, Leng SX. Frailty syndrome: an overview. Clin Interv Aging. 2014;9:433-441.

8. Lang P, Mitchell W, Lapenna A, Pitts D, Aspinall R. Immunological pathogenesis of main age-related diseases and frailty: role of immunosenescence. Eur Geriatr Med. 2010;1(2):112-121.

9. Crentsil V. Mechanistic contribution of carnitine deficiency to geriatric frailty. Ageing Res Rev. 2010;9(3):265-268.

10. Mocchegiani E, Corsonello A, Lattanzio F. Frailty, ageing and inflammation: reality and perspectives. Biogerontology. 2010;11(5):523-525.

11. Malaguarnera M. Carnitine derivatives: clinical usefulness. Curr Opin Gastroenterol. 2012;28(2):166-176.
12. Stephens FB, Constantin-Teodosiu D, Greenhaff PL. New insights concerning the role of carnitine in the regulation of fuel metabolism in skeletal muscle. J Physiol. 2007;581(2):431-444.

13. Ribas GS, Vargas CR, Wajner M. L-carnitine supplementation as a potential antioxidant therapy for inherited neurometabolic disorders. Gene. 2014;533(2):469-476.

14. Gramignano G, Lusso MR, Madeddu C, et al. Efficacy of 1-carnitine administration on fatigue, nutritional status, oxidative stress, and related quality of life in 12 advanced cancer patients undergoing anticancer therapy. Nutrition. 2006;22(2):136-145.

15. Busquets S, Serpe R, Toledo M, et al. 1-Carnitine: An adequate supplement for a multi-targeted anti-wasting therapy in cancer. Clin Nutr. 2012; 31(6):889-895.

16. Cruciani RA, Dvorkin E, Homel P, et al. Safety, tolerability and symptom outcomes associated with L-carnitine supplementation in patients with cancer, fatigue, and carnitine deficiency: a phase I/II study. J Pain Symptom Manage. 2006;32(6):551-559.

17. Cruciani RA, Zhang JJ, Manola J, Cella D, Ansari B, Fisch MJ. $\mathrm{L}$-carnitine supplementation for the management of fatigue in patients with cancer: an eastern cooperative oncology group phase III, randomized, double-blind, placebo-controlled trial. J Clin Oncol. 2012;30(31): 3864-3869.

18. De Simone C, Tzantzoglou S, Famularo G, et al. High dose L-carnitine improves immunologic and metabolic parameters in AIDS patients. Immunopharmacol Immunotoxicol. 1993;15(1):1-12.

19. Moretti S, Famularo G, Marcellini S, et al. L-carnitine reduces lymphocyte apoptosis and oxidant stress in HIV-1-infected subjects treated with zidovudine and didanosine. Antioxid Redox Signal. 2002;4(3): 391-403.

20. Siami G, Clinton M, Mrak R, Griffis J, Stone W. Evaluation of the effect of intravenous L-carnitine therapy on function, structure and fatty acid metabolism of skeletal muscle in patients receiving chronic hemodialysis. Nephron. 1991;57(3):306-313.

21. Malaguarnera M, Cammalleri L, Gargante MP, Vacante M, Colonna V, Motta M. L-Carnitine treatment reduces severity of physical and mental fatigue and increases cognitive functions in centenarians: a randomized and controlled clinical trial. Am J Clin Nutr. 2007;86(6):1738-1744.

22. Malaguarnera M, Gargante MP, Cristaldi E, et al. Acetyl L-carnitine (ALC) treatment in elderly patients with fatigue. Arch Gerontol Geriatr. 2008;46(2):181-190.

23. Bersani G, Meco G, Denaro A, et al. 1-Acetylcarnitine in dysthymic disorder in elderly patients: A double-blind, multicenter, controlled randomized study vs. fluoxetine. Eur Neuropsychopharmacol. 2013;23(10): 1219-1225.

24. Chan Y. Randomised controlled trials (RCTs)-sample size: the magic number? Singapore Med J. 2003;44(4):172-174.

25. Rondanelli M, Opizzi A, Antoniello N, et al. Effect of essential amino acid supplementation on quality of life, amino acid profile and strength in institutionalized elderly patients. Clin Nutr. 2011;30(5):571-577.

26. Shahar S, Omar A, Vanoh D, et al. Approaches in methodology for population-based longitudinal study on neuroprotective model for healthy longevity (TUA) among Malaysian older adults. Aging Clin Exp Res. Epub 2015 Dec 15.

27. Fried LP, Tangen CM, Walston J, et al. Frailty in older adults evidence for a phenotype. J Gerontol A Biol Sci Med Sci. 2001;56(3): M146-M157.

28. Rockwood K, Andrew M, Mitnitski A. A comparison of two approaches to measuring frailty in elderly people. $J$ Gerontol A Biol Sci Med Sci. 2007;62(7):738-743.

29. De Vries N, Staal J, van Ravensberg C, Hobbelen J, Olde Rikkert M, Nijhuis-Van der Sanden M. Outcome instruments to measure frailty: a systematic review. Ageing Res Rev. 2011;10(1):104-114.

30. Searle SD, Mitnitski A, Gahbauer EA, Gill TM, Rockwood K. A standard procedure for creating a frailty index. BMC Geriatr. 2008;8(1):24.

31. Washburn RA, Smith KW, Jette AM, Janney CA. The physical activity scale for the elderly (PASE): Development and evaluation. $J$ Clin Epidemiol. 1993;46(2):153-162. 
32. Ngai SP, Cheung RT, Lam PL, Chiu JK, Fung EY. Validation and reliability of the physical activity scale for the elderly in Chinese population. J Rehabil Med. 2012;44(5):462-465.

33. Lee RD, Nieman DC. Nutritional Assessment. St Louis: McGraw-Hill Higher Education; 2007.

34. Folstein MF, Folstein SE, McHugh PR. "Mini-mental state": a practical method for grading the cognitive state of patients for the clinician. J Psychiatr Res. 1975;12(3):189-198.

35. Ibrahim NM, Shohaimi S, Chong HT, et al. Validation study of the mini-mental state examination in a Malay-speaking elderly population in Malaysia. Dement Geriatr Cogn Disord. 2009;27(3):247-253.

36. Bohannon RW, Schaubert KL. Test-retest reliability of grip-strength measures obtained over a 12-week interval from community-dwelling elders. J Hand Ther. 2005;18(4):426-428.

37. Johansson KM, Adolfsson LE. Intraobserver and interobserver reliability for the strength test in the Constant-Murley shoulder assessment. J Shoulder Elbow Surg. 2005;14(3):273-278.

38. Jones CJ, Rikli RE, Beam WC. A 30-s chair-stand test as a measure of lower body strength in community-residing older adults. Res $Q$ Exerc Sport. 1999;70(2):113-119.

39. Podsiadlo D, Richardson S. The timed "Up \& Go": a test of basic functional mobility for frail elderly persons. J Am Geriatr Soc. 1991;39(2): 142-148.

40. Peters DM, Fritz SL, Krotish DE. Assessing the reliability and validity of a shorter walk test compared with the 10-meter walk test for measurements of gait speed in healthy, older adults. J Geriatr Phys Ther. 2013;36(1):24-30.

41. Katz S, Ford AB, Moskowitz RW, et al. Studies of illness in the aged: the index of ADL: a standardized measure of biological and psychosocial function. Jama. 1963;185(12):914-919.
42. Prieto JA, Andrade F, Aldámiz-Echevarría L, Sanjurjo P. Determination of free and total carnitine in plasma by an enzymatic reaction and spectrophotometric quantitation spectrophotometric determination of carnitine. Clin Biochem. 2006;39(10):1022-1027.

43. Hoppel $\mathrm{C}$. The role of carnitine in normal and altered fatty acid metabolism. Am J Kidney Dis. 2003;41:S4-S12.

44. Silvério R, Laviano A, Rossi Fanelli F, Seelaender M. L-carnitine and cancer cachexia: clinical and experimental aspects. J Cachexia Sarcopenia Muscle. 2011;2(1):37-44.

45. Cavallini G, Caracciolo S, Vitali G, Modenini F, Biagiotti G. Carnitine versus androgen administration in the treatment of sexual dysfunction, depressed mood, and fatigue associated with male aging. Urology. 2004;63(4):641-646.

46. Pertosa G, Grandaliano G, Simone S, Soccio M, Schena FP. Inflammation and carnitine in hemodialysis patients. J Ren Nutr. 2005;15(1): $8-12$.

47. Duranay M, Akay H, Yilmaz FM, Şeneş M, Tekeli N, Yücel D. Effects of L-carnitine infusions on inflammatory and nutritional markers in haemodialysis patients. Nephrol Dial Transplant. 2006;21(11):3211-3214.

48. Suchitra M, Ashalatha V, Sailaja E, et al. The effect of 1-carnitine supplementation on lipid parameters, inflammatory and nutritional markers in maintenance hemodialysis patients. Saudi J Kidney Dis Transpl. 2011;22(6):1155.

49. Shakeri A, Tabibi H, Hedayati M. Effects of 1-carnitine supplement on serum inflammatory cytokines, C-reactive protein, lipoprotein (a), and oxidative stress in hemodialysis patients with Lp (a) hyperlipoproteinemia. Hemodial Int. 2010;14(4):498-504.

50. Calabrese V, Stella AMG, Calvani M, Butterfield DA. Acetylcarnitine and cellular stress response: roles in nutritional redox homeostasis and regulation of longevity genes. J Nutr Biochem. 2006;17(2):73-88.
Clinical Interventions in Aging

\section{Publish your work in this journal}

Clinical Interventions in Aging is an international, peer-reviewed journal focusing on evidence-based reports on the value or lack thereof of treatments intended to prevent or delay the onset of maladaptive correlates of aging in human beings. This journal is indexed on PubMed Central, MedLine,

\section{Dovepress}

CAS, Scopus and the Elsevier Bibliographic databases. The manuscript management system is completely online and includes a very quick and fair peer-review system, which is all easy to use. Visit http://www.dovepress. com/testimonials.php to read real quotes from published authors. 